rium trajectories predicted from our experimental data were different from the actual trajectories because of low stiffness. This result indicates that not only stiffness needs to be taken into account in movement planning but also the dynamics of the controlled object, even if the planning output is an equilibrium point trajectory. I would like to emphasize that a similar planning complexity exists when obtaining a movement plan by means of computing the inverse dynamics of the controlled object. Another consideration about what is planned in the cerebellum comes from the clinical studies. The fact that cerebellar patients cannot execute movements naturally implies that they lost an important ingredient of motor control. If it were only stiffness control, an equilibrium point trajectory plan which, for this argument, is hypothesized to take place in another brain structure, would actually be able to compensate for the loss of stiffness control by changing the trajectory plans to take the cerebellar deficit into account. The lack of this effect seems to suggest that the cerebellum codes an important element of the final motor command.

Neural activities in the cerebellum further support this idea. The observation of the P-cell activities independent of movement direction (Fortier et al. 1993) could be interpreted as P-cell inhibition of excessive or inappropriate inputs in all irrelevant movement directions to the cerebellar nucleus. The preferred direction of $\mathrm{P}$-cells contributes to produce the muscle tension imbalance which generates torque patterns for smooth coordinated movements. Actually, many P-cells were directionally activated during single-joint movements (Frysinger et al. 1984), and $P$-cell firing frequencies were predicted from smooth eye movements elicited by large visual scene (Shidara et al. 1993).

From those discussions, my interpretation of a motion control strategy is: (1) Learners initially try to imitate the kinematics of a demonstrated skillful movement. Their stiffness might be high enough to reduce the unknown effects of dynamics of limbs and the external environment, as shown in the simulation of multi-joint movements (Flash 1987). Unskilled movements would still be clumsy and would easily be exhausting. (2) As the brain acquires internal models of the controlled object, stiffness decreases in an optimization process to avoid fatigue or to achieve some targets. The stiffness would be controlled according to the constraints of the external environment and the requested tasks.

From this discussion, the role of the cerebellum in movement control is that it learns muscle activation patterns for smooth, accurate, and effortless movements, and that it controls not only joint stiffness but also torques, as generated by muscle tension imbalance. In any case, stiffness is important information in biological motor control, as described in SMITH's target article. To understand the control mechanism, it might be necessary to build a model that can predict the stiffness from important factors such as motion purpose, strategies, and external environmental conditions.

\section{What can and what cannot be adjusted in the movement patterns of cerebellar patients?}

\section{Patrick Haggard \\ Department of Psychology, University of College London, London WC1E 6BT, England.p.haggard@ucl.ac.uk}

Abstract: This commentary reviews the case of a patient who could alter the coordination of her prehensile movements when removal of visual feedback reduced her kinetic tremor, but could not coordinate her hand aperture with her hand transport within a single movement. This suggests a dissociation between different subtypes of cerebellar context-response linkage, rather than a single, general association function. [TIACII]

How general is the cerebellar context-response linkage mechanism proposed in THACI's target article? In a recent study of coordinated reaching and grasping movements in a unilateral cerebellar patient (Haggard et al. 1994), we found a dissociation between two different kinds of motor adjustment, one which could be made following cerebellar lesion, and one which could not. This reply uses this dissociation to clarify what kinds of motor learning the cerebellum may provide.

Our patient, $\mathrm{KA}$, was left with profound intention tremor and hypermetria, largely confined to the right arm, following surgical removal of an ependymoma of the IVth ventricle. She also had hypotonia of the right limbs, and a profoundly ataxic gait. We tested the patient's ability to make coordinated reaching and grasping movements. In normal prehension (Jeannerod 1981), the hand preshapes to grasp the object in a way which is tightly spatially coupled with hand's approach to the target (Haggard \& Wing 1995). This normal pattern was seen in our patient's unaffected left hand. The right hand opened much wider than the left, to compensate for the profound intention tremor of the right arm: a greater hand aperture gave the patient a greater chance of grasping the target object.

We then compared maximum hand aperture during reaches made in normal lighting conditions, and in complete darkness. The normal left hand opened wider in the no vision condition, replicating the finding of Wing et al. (1986). The affected right hand, in contrast, showed an interesting pattern of movement in the no vision condition: there was a significant decrease in the severity of the intention tremor in the no vision condition (Beppu et al. 1987). Therefore, removing visual feedback increased the level of certainty that the patient could have about movements of her right arm, even though it decreased the level of certainty she could have about movements of her normal left arm. The maximum right hand aperture was accordingly reduced in the no vision condition. The patient thus has a preserved ability to monitor the effects of the movement context on reaching accuracy, and adjust hand aperture accordingly. Therefore, the cerebellum does not seem to be required in order to make functional strategic adjustments to motor patterns.

In contrast to the successful adjustments, KA could not coordinate opening and closing of the hand with the forward movement of the arm in any given trial. In normals, the motor system controls hand aperture so as to keep its spatial relation to hand transport constant (Haggard \& Wing 1995). Therefore, plots of hand aperture against hand transport on repeated trials normally show a decrease in variability as the hand approaches the target, as hand aperture is adjusted so that it bears the appropriate spatial relation to hand transport. In this sense, the hand transport can be treated as a context, and coordination as a linkage which selects an appropriate instantaneous hand aperture for that context.

We observed a significant decrease in variability of the hand aperture against hand transport plot as movements progressed from start to target for KA's normal left hand. However, a significant increase in variability was found for the affected right hand. This pattern was found for movements both with and without vision (Fig. 1). Therefore, cerebellar damage does affect the context-response linkage, or coordination, between the hand transport and hand aperture components of prehension.

Our patient, then, could adjust her movement patterns to compensate for a change environmental context, but could not adjust them within a single trial to preserve an appropriate coordination of an action as a whole. This result suggests that the context-response mechanism in the cerebellum is not entirely general: some forms of linkage are lost following cerebellar damage while others are preserved. What informational features of the second, within-trial, form of response adjustment make it specifically vulnerable to cerebellar damage? In prehension, an important distinction between these two kinds of adjustment is that the first (modulating maximum aperture according to environmental conditions) relies on a strategy arising from knowledge of results of previous movements, and anticipation of the likely success of forthcoming movements. The spatial coordina- 
Loft hand: Wilh vision

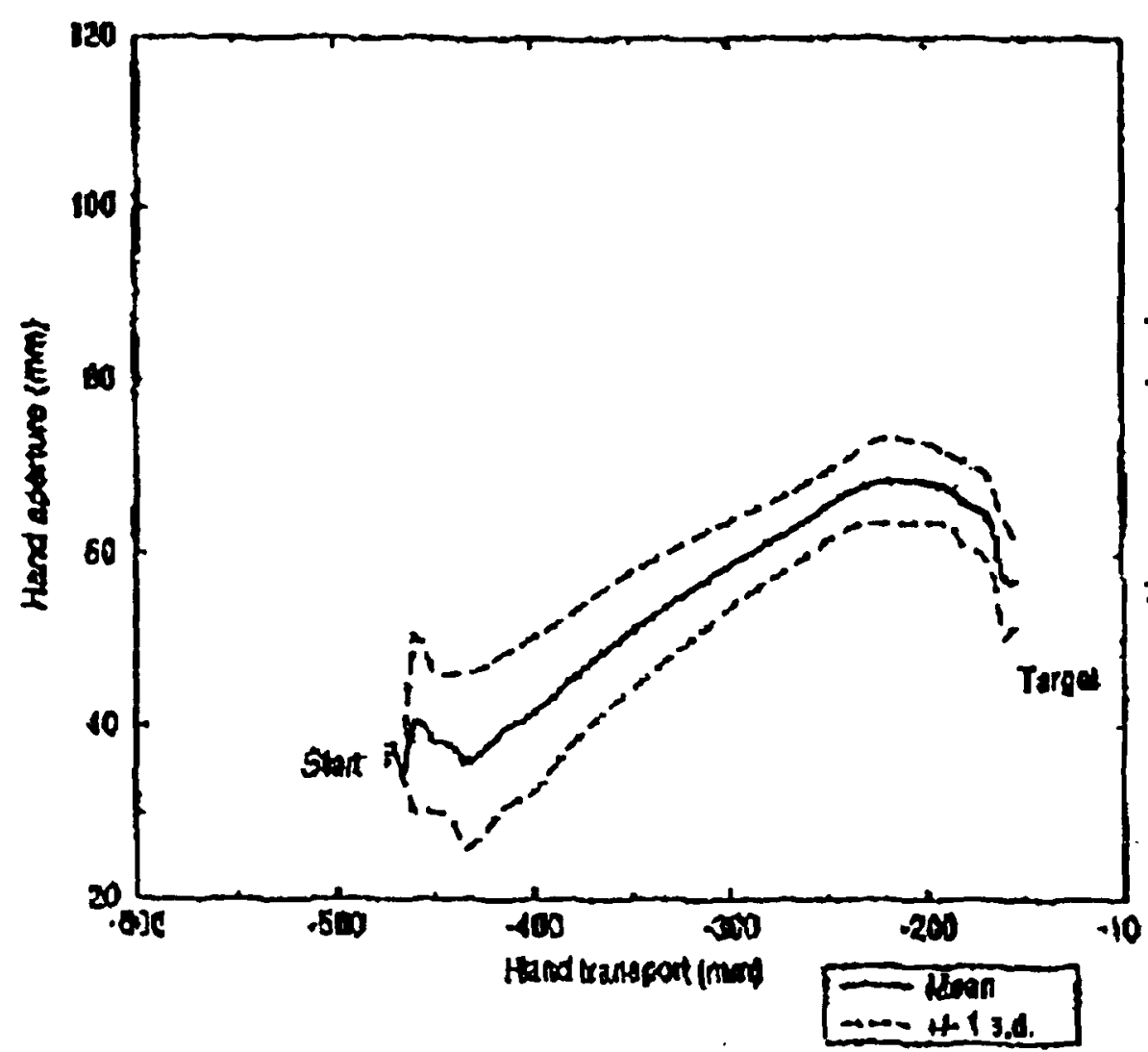

Loff hand: No vision

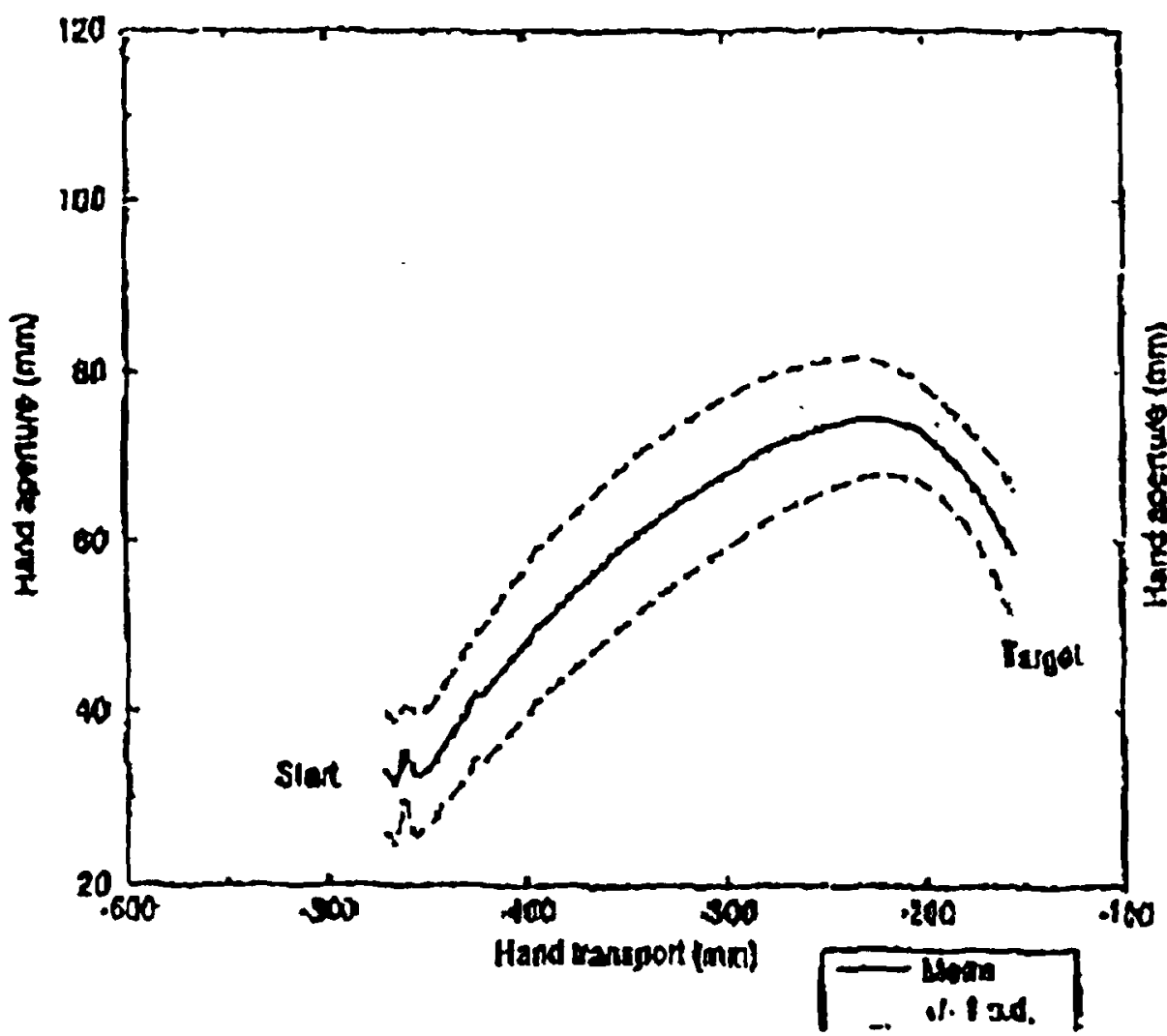

Right hand: With vision

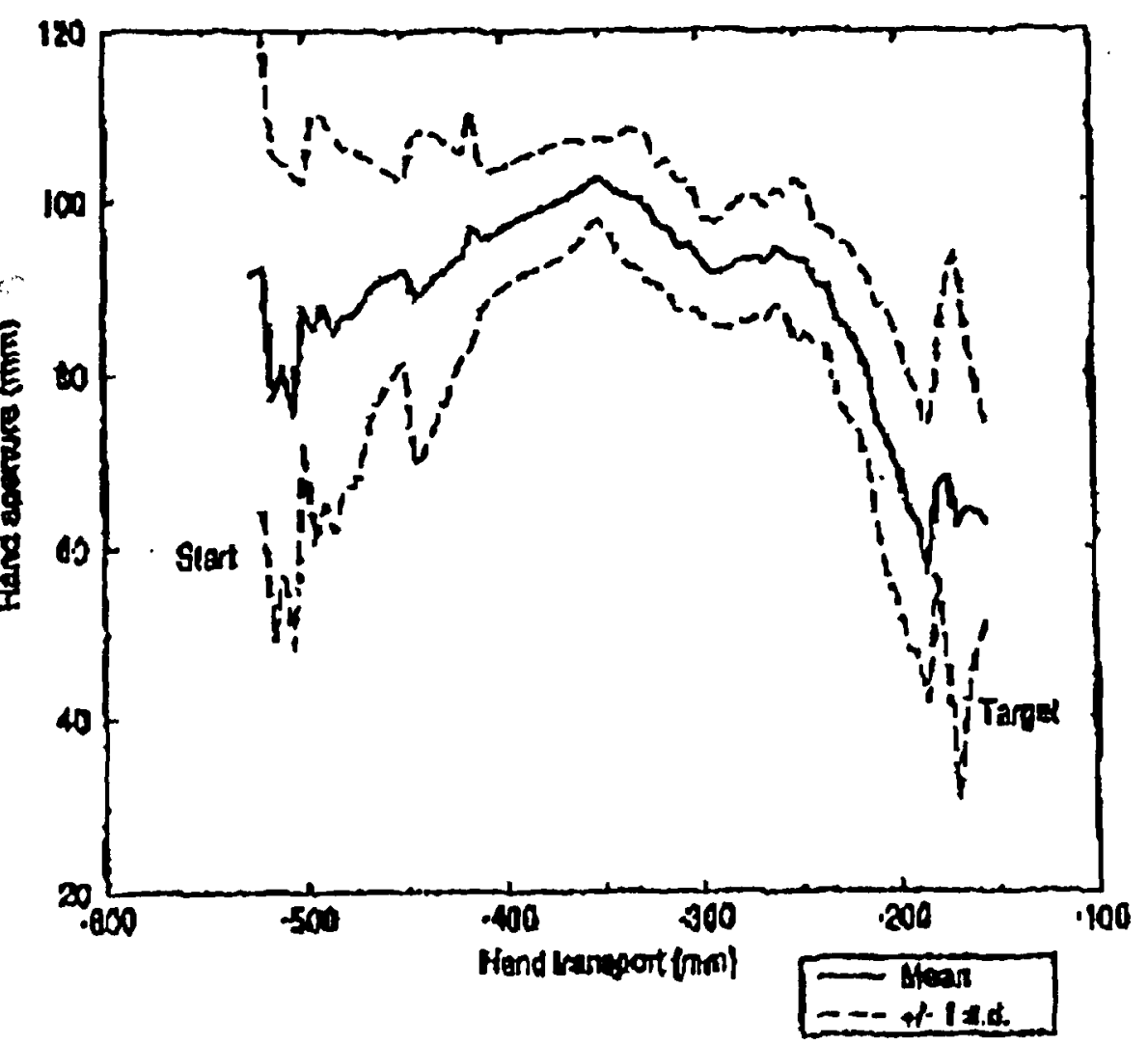

Aight trand: No vision

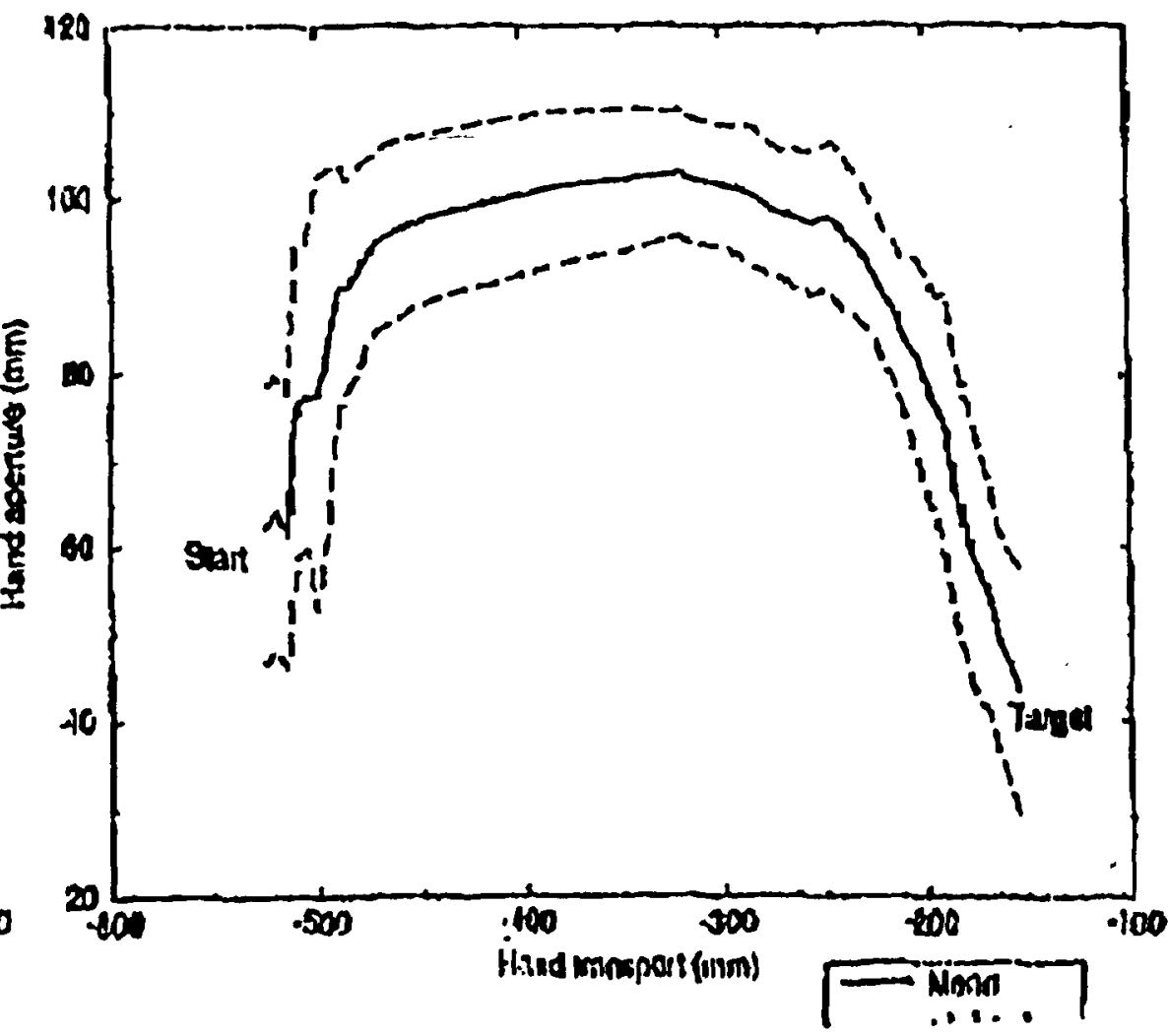

Figure 1 (Haggard). Mean (solid line) $+1-1$ standard deviation (dashed lines) of the spatial relation between hand aperture (fingerthumb distance) and hand transport (thumb position along start-target axis) for left (unaffected) and right (affected) hands, with and without visual feedback, in patient KA. The target is located at $-150 \mathrm{~mm}$ on the abscissa. The vertical distance between the solid and dashed lines gives an index of the regularity of repeated movements at each stage of hand transport. Reprinted from Neuropsychologia (1994), with kind permission from Elsevier.

tion of aperture and transport in a single trial in contrast, is not based on strategies and knowledge of results, but instead resembles a servo system.

In this view, the cerebellum could usefully contribute to any behavior or neural operation which required rapid adjustment of control parameters to maintain an optimal motor output. But the cerebellum is not a necessary element in behaviors which involve prior heuristic selection of one response from an extensive range of options. If the motor function of the cerebellum is modeled as "context-response linkage," then the cerebellar linkage seems designed for optimizing ongoing responses, rather than planning or selecting "smart" movement patterns in advance. 